\title{
Effects of Fermentation under Enriched Oxygen Atmosphere on Clonal Black Tea Aroma Complex
}

\author{
P. Okinda OwUOR and Martin OBANDA \\ Tea Research Foundation of Kenya, P.O. Box 820, KERICHO, Kenya
}

Received September 2, 1997; Accepted November 10, 1997

\begin{abstract}
Enriching the atmosphere of fermenting "dhool" (macerated leaf) with oxygen led to faster production of some black tea volatile flavor compounds (VFC). There was a general rise in the total amounts of the VFC with long fermentation duration irrespective of whether it was done under enriched oxygen atmosphere or ambient air. The rise was, however faster under enriched oxygen atmosphere than under ambient air. The rise was also faster in the group I VFC than the group II VFC leading to a general decline in the Flavor Index with long fermentation durations. For every fermentation duration, the sum of group I VFC was higher under enriched oxygen atmosphere than under ambient air fermentation. Such variations were only marginal in the group II VFC. As a result, fermentation under enriched oxygen atmosphere reduced the aroma quality of black tea despite increasing the total amounts of VFC. Generally, the VFC which are oxidative products of amino acids and unsaturated fatty acids degradation increased with fermentation durations, while the major terpene alcohols declined under both ambient air and enriched oxygen atmosphere fermentation conditions.
\end{abstract}

Keywords: Black tea aroma, fermentation under enriched oxygen atmosphere, volatile flavor compounds, quality

Fermentation in the production of black tea from the soft tender shoots of Camellia sinensis (L.) Kuntze is generally associated with oxidative transformation of polyphenols mainly catechins to theaflavins and thearubigins (Roberts \& Wood, 1950). The process is initiated by polyphenol oxidase enzyme which is abundant in the chloroplast of fresh tea leaves (Gregory \& Bendall, 1966; Hilton, 1974) and requires the presence of oxygen (Roberts \& Wood, 1951; Horner \& Durckheimer, 1959), normally supplied by blowing ambient air through the "dhool" (macerated tea leaf). Inadequate supply of the oxygen leads to dull liquors and dark infusions (Werkhoven, 1974). This is normally attributed to a change in the reaction path leading to the formation of thearubigins at the expense of theaflavins (Werkhoven, 1974; Harler, 1963). The effects of the supply of excess oxygen on the formation of these compounds were recently reported (Owuor \& Obanda 1998).

However, there are also other oxidative reactions concurrently taking place during the fermentation phase of black tea processing (Owuor et al., 1994). The amino acids undergo oxidation via the Strecker degradation process leading into the release of aldehydes (Co \& Sanderson, 1970; Saijo \& Takeo, 1970a). Valine, leucine, isoleucine (Co \& Sanderson, 1970) and phenylalanine (Co \& Sanderson, 1970; Saijo \& Takeo, 1970a, b) are thus converted to 2-methylpropanal, 3-methylbutanal, 2-methylbutanal and phenylacetaldehyde, respectively. These reactions are catalyzed by polyphenol oxidase or peroxidase (Saijo \& Takeo, 1970a, b) in the presence of oxygen and catechins (Robinson \& Owuor, 1991). The formed aldehydes can be further oxidized to carboxylic acids (Robinson \& Owuor, 1991).

At the same time, the unsaturated free fatty acids in the tea leaves are also oxidized to mainly $\mathrm{C}_{6}$ aldehydes during fermentation (Hatanaka et al. 1987). Linoleic and linolenic acids are, for example, oxidized to hexanal and Z-3-hexenal which isomerises to $E$-2-hexenal (Hatanaka et al. 1987; 1976). The oxidation takes place in the presence of lipoxygenase (Takeo \& Tsushida, 1980) and oxygen (Robinson \& Owuor, 1991). The produced aldehydes can either be further oxidized to carboxylic acids or reduced to primary alcohols (Robinson \& Owuor, 1991), all of which are volatile flavor compounds (VFC) which contribute to the aroma quality of black tea.

The terpene glycosides levels in green tea leaves also undergo changes during black tea processing through a hydrolytic process (Takeo, 1981) leading to the variations in the amounts of terpene alcohols in the tea (Robinson \& Owuor, 1991). The role of oxygen in these changes is less obvious. However, from these observations, it is noted that the oxygen supply is an important requirement for the formation of many black tea aroma compounds.

At present, oxygen is supplied to the fermenting "dhool" (macerated leaves) by blowing ambient air through it during fermentation. The effect of varying the quantity of oxygen on the relative formation of the VFC is not clearly understood. It is, therefore, unclear whether or not the supplied oxygen in ambient air is adequate or not. This study was done to assess the effect of enriched oxygen atmosphere during black tea fermentation on the production of the VFC and its resultant effects on the aroma quality of black tea.

\section{Experimental}

Young tender shoots of clones $6 / 8$ and $31 / 11$ were obtained from a commercial field at the Timbilil Estate of the Tea Research Foundation of Kenya, situated at $2180 \mathrm{~m}$ above mean sea level, at latitude $0^{\circ} 15^{\prime}$ South and longitude $35^{\circ} 21^{\prime}$ East. The fields were commercially managed and had received 
fertilizer as NPKS 25:5:5:5 at $150 \mathrm{~kg} \mathrm{~N}$ ha/year applied in one dose. The leaf was hand plucked to conform to the normal plucking standard with over $70 \%$ acceptable leaves of up to two leaves and a bud.

After plucking, the leaf was withered to achieve $70-72 \%$ wither (Owuor \& Orchard, 1989) in a controlled environment (Teacraft Ltd.) at $22^{\circ} \mathrm{C}$ dry bulb and $20^{\circ} \mathrm{C}$ wet bulb temperature, for $14 \mathrm{~h}$. The leaf in $600 \mathrm{~g}$ batches was then macerated using miniature Crush, Tear and Curl (CTC) rollers and then fermented for 50, 90, and $130 \mathrm{~min}$ under ambient conditions or enriched oxygen atmosphere. Oxygen enrichment was achieved by placing the macerated "dhool" in a polyethylene bag, then removing the air under vacuum and refilling the bag with oxygen, the operation was repeated three times.

At the end of the set fermentation time, the "dhool" was fired (dried) using a miniature fluid bed dryer (Teacraft). The manufacture of each clone was repeated on three different occasions, and each repeat was used as a replicate. The unsorted black teas were subjected to VFC analysis without sorting.

Analysis of the volatile flavor compounds (VFC) Modified simultaneous steam distillation extraction (SDE, water/diethyl ether) was used to extract the VFC (Likens \& Nickerson, 1964) as earlier outlined (Baruah et al. 1986; Owuor et al., 1986a) using cumene as an internal standard. For every $1 \mathrm{~g}$ black tea, $3.75 \mu \mathrm{g}$ cumene was used as internal standard.

The dried (anhydrous $\mathrm{Na}_{2} \mathrm{SO}_{4}$ ) ether mixture was concentrated to about $100 \mu \mathrm{l}$ and then subjected to gas chromatography (GC) and GC-mass spectrometry (GC-MS) analysis as outlined by Baruah et al. (1986). GC was performed on a Varian 3300 model gas chromatograph equipped with flame ionized detector, while GC-MS was done on a Hitachi model $663 \mathrm{GC}$ coupled with a Hitachi M.80A and M.003 mass spectrometer. Peak identifications were made by comparing retention times with those of the authentic samples and confirmed by GC-MS. The quantities of the compounds are expressed as the ratio of the peak area to that of the internal standard. The aroma quality was quantified by the Flavor Index as the ratio of the sum of the gas chromatographic peak areas of the compounds imparting sweet floral aroma, i.e., group II VFC, to those imparting green grassy smell, i.e., group I VFC (Owuor, 1992).

\section{Results and Discussion}

Black tea contains many VFC. Some the these VFC impart green, grassy undesirable aroma to black tea. These compounds are classified as group I VFC. However, there are also those imparting desirable sweet flowery aroma to black tea, which are grouped as group II VFC. The ratio of the sum of the gas chromatographic peak areas of the VFC with desirable flowery aroma (group II) to those with undesirable green grassy aroma (group I) is known as the Flavor Index (Owuor et al., 1986a; Owuor 1992). Aroma is an important quality parameter for some black teas and various indices have been used to evaluate the aroma quality of black teas in different parts of the world (Wickremasinghe et al., 1973; Yamanishi et al., 1978; 1989; Owuor et al., 1986a; Baruah et al., 1986). For Kenyan teas, a better relationship has been obtained between sensory evaluation and the Flavor Index (Owuor et al., 1988; Owuor, 1992) than the plain black tea quality parameters (Owuor et al., 1986b; 1987a). In this study, the Index was used to quantify the effects of enriched oxygen atmosphere and varying fermentation durations on the aroma quality of the resultant black tea.

In a previous study, it was demonstrated that only minute amounts of developed VFC are retained in black tea, as most of the VFC are lost during the firing stage of black tea processing (Owuor et al., 1994). However, despite the loss of high amounts of the VFC, the amount retained in the black tea is proportional to the original amounts, provided firing conditions are optimized (Owuor et al., 1987b). Thus, the variations caused by processing conditions and/or agronomic practices are preserved/maintained in the resultant black teas. For example, fermentation under ambient conditions was demonstrated to increase the total amount of VFC, especially the group I VFC and this led to the decline in the Flavor Index with long fermentation durations (Owuor et al., 1994). Similar results were recorded in this study for the two clones used (Table 1). These results confirm the earlier observations that for the processing of aromatic black tea, short fermentation durations are ideal (Owuor et al., 1994).

Similar to fermentation under ambient air, there was a rise in the total amounts of the VFC with an increase in fermentation duration under enriched oxygen atmosphere (Table 1). The rise in the development of the group I VFC was much faster than that for group II VFC under enriched oxygen atmosphere than under ambient air fermentation. Also, the amount of group I VFC was much higher than group II VFC under enriched oxygen atmosphere than under ambient air fermentation. Thus, the Flavor Index of the black tea produced under enriched oxygen atmosphere was lower than that of the black tea produced under ambient air at equivalent fermentation duration.

The group I VFC were dominated by the products of unsaturated fatty acids oxidation, especially $E$-2-hexenal, hexanal, $Z$-3-hexenal, nonanal and their reduction products, i.e., the respective primary alcohols. The products of amino acids degradation, i.e. 2-methylbutanal and pentanal were also abundant. Some of the amino acids degradation products were, however, in the group II VFC fraction, these included benzaldehyde and phenylacetaldehyde. The compounds, as expected, increased with longer fermentation duration. However, their rise under enriched oxygen atmosphere was faster than under ambient air fermentation. This shows that more complete degradation of the precursor compounds are observed under enriched oxygen atmosphere than under ambient air fermentation. However, the situation only leads to the production of lower quality black teas as these compounds are mainly the group I VFC, whose increase is deleterious to black tea quality (Owuor, 1992; Owuor et al., 1988).

Terpene alcohols are an important quality parameter in black tea aroma (Baruah et al., 1986; Owuor, 1992) and are produced from terpene glycosides by a hydrolytic process (Takeo, 1981). This process requires the activity of the enzyme hydrolase (Robinson \& Owuor, 1991). Increasing fermentation duration led to lower values of the dominant monoter- 
Table 1. Effect of enriched oxygen atmosphere on the formation of volatile flavor compounds in black tea.

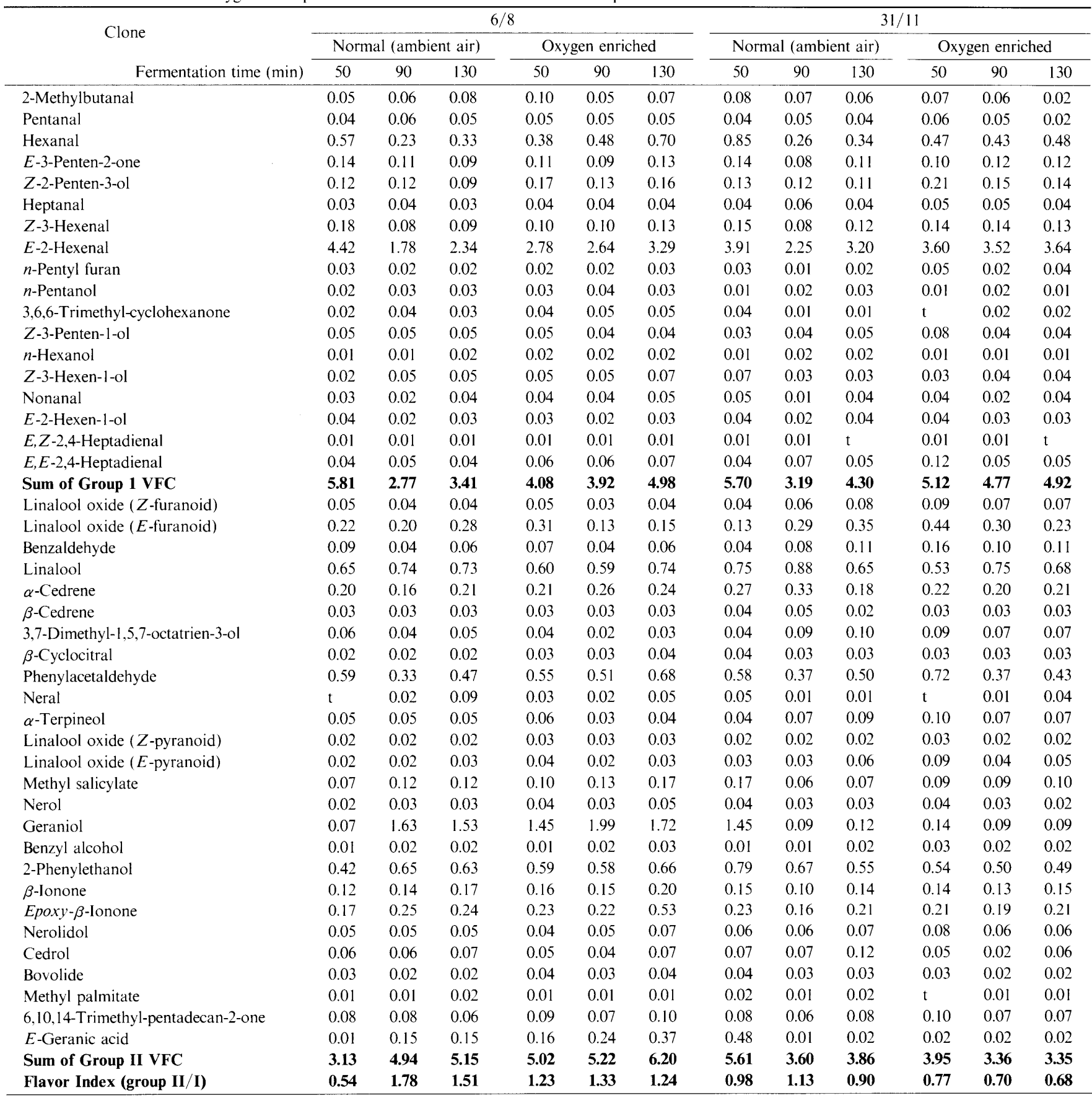

$\mathrm{t}=$ trace

penes, i.e., linalool and geraniol. This is similar to the results observed in a previous study (Owuor et al., 1994). However, the decline was greater under enriched oxygen atmosphere than under ambient air for geraniol in clone $6 / 8$. The exact involvement of oxygen in the hydrolytic process is not clearly understood, but it is speculated that oxygen could be inhibiting the hydrolysis. Such speculation, however, needs to be demonstrated with experimentation. Alternatively, under enriched oxygen, the geraniol was further oxidized to $E$ geranic acid. Indeed, the levels of $E$-geranic acid were higher and showed faster increase in clone $6 / 8$ when fermentation was carried out under enriched oxygen atmosphere. The linalool oxides which are oxidative products of linalool, however, did not show a similar increase.

There were many VFC, such $\alpha$-cedrene, $\beta$-cedrene, cedrol, neral, nerol, 3,7-dimethyl-1,5,7-octatrien-3-ol, $\beta$-cyclocitral, $\alpha$-terpineol, methyl salicylate, ionones, nerolidol, bovolide, 6,10,14-trimethylpentandecan-2-one, etc. whose levels were not affected by the fermentation atmosphere. Their formation did not appear to require oxygen, most of them were either primary products from the fresh tea leaves or pyrolytic products of carotenes degradation during firing (Robinson \& Owuor, 1991).

The results demonstrate that the present system where 
oxygen is not augmented to fermenting "dhool" is beneficial to black tea quality despite decreasing the total VFC produced. Indeed, denying the black tea excessive oxygen by reducing the amounts of oxygen in the ambient air could improve its aroma quality.

Acknowledgment Data collection by the staff of the Chemistry Department, Tea Research Foundation of Kenya (TRFK) is gratefully acknowledged. This paper is presented with the kind permission of the Director, TRFK.

\section{References}

Baruah, S., Hazarika, M., Mahanta, P.K., Horita, H. and Murai, T. (1986). The effect of plucking intervals on the chemical constituents of CTC black teas. Agric. Biol. Chem., 50, 1039-1041.

Co, H. and Sanderson, G.W. (1970). Biochemistry of tea fermentation. Conversion of amino acids to black tea aroma constituents. J. Food Sci., 35, 160- 164.

Gregory, R.P.F. and Bendall, D.S. (1966). Purification and properties of polyphenol oxidase. Biochem. J., 101, 569-581.

Harler, C.R. (1963). "Tea Manufacture," Oxford University Press.

Hatanaka, A., Kajiwara, T. and Sekiya, J. (1976). Biosynthesis of trans-2-hexenal in chloroplasts from Thea sinensis. Phytochemistry, 15, $1125-1126$.

Hatanaka, A., Kajiwara, T. and Sekiya, J. (1987). Enzymic oxygenative-cleavage reaction of linolenic acid in leaveschloroplastic lipoxygenase and fatty acid hydroperoxide lyase in tea leaves. In "Metabolism, Structure and Function of Plant Lipids," ed. by P.K. Stumpf. J.B. Mudd. and W.B. Nes, Plenum Publishing Corporation, New York, pp. 391-398.

Hilton, P.J. (1974). The effect of shade upon the chemical composition of the flush of tea Camellia sinensis. Trop. Sci., 16, 15-23.

Horner, L. and Durckheimer, W. (1959). o-Quinones from catechol derivatives. Z. Naturforsch., 146, 741-746.

Kobayashi, A., Kawamura, M., Yamamoto, Y., Shimizu, K., Kubota, K. and Yamanishi, T. (1988). Methyl epijasmonate in the essential oil of tea. Agric. Biol. Chem., 52, 2299-2303.

Likens, L.S. and Nickerson. C.B. (1964). Detection of certain hop oil constituents in brewing products. Proc. Am. Soc. Brewing Chem., 5-13.

Owuor, P.O. (1992). Comparison of gas chromatographic volatile profiling methods for assessing the flavor quality of Kenyan black teas. J. Sci. Food Agric., 59, 189-197.

Owuor, P.O., Horita, H., Tsushida, T. and Murai, T. (1986a). Comparison of the chemical compositions of black teas from main black tea producing parts of the world. Tea, 7, 71-78.

Owuor, P.O. and Obanda, M. (1998). Influence of enriched oxygen atmosphere during fermentation on plain clonal black tea quality parameters development. Trop. Sci. (in press).

Owuor, P.O., Obanda, M.A. and Wanyiera, J.O. (1987b). The effect of drier type and grading on chemical quality parameters of black tea. Tea, 8, 73-80.

Owuor, P.O. and Orchard, J.E. (1989). The effect of physical wither on the chemical composition of black tea. Tea, 10, 47-52.

Owuor, P.O., Orchard, J.E. and McDowell, I.J. (1994). Changes in the quality parameters of clonal tea due to fermentation time. $J$. Sci. Food Agric., 64, 319-326.

Owuor, P.O., Othieno, C.O. and Reeves, S.G. (1987a). The Kenya position on theaflavins in tea. J. Food Agric., 1, 185-186.

Owuor, P.O., Reeves, S.G. and Wanyoko, J.K. (1986b). Correlation of theaflavins contents and valuation of Kenyan black teas. J. Sci. Food Agric., 37, 507-513.

Owuor, P.O., Tsushida, T., Horita, H. and Murai, T. (1988). Effects of geographical area of production on the composition of volatile flavor compounds in Kenyan clonal black CTC teas. Exp. Agric., 24, 227-235.

Roberts, E.A.H. and Wood, D.J. (1950). The fermentation process in tea manufacture-Oxidation of substrates by oxidases. Biochem. J., 47, 175-186.

Roberts, E.A.H. and Wood, D.J. (1951). Oxidation of anthoxanthins by tea oxidase. Nature (London), 167, 608 .

Robinson, J.M. and Owuor, P.O. (1991). Tea aroma. In "Tea, Cultivation to Consumption." ed. by K.C. Willson and M.N. Clifford, Chapman and Hall, London, Chapter 18, pp. 603-648.

Saijo, R. and Takeo, T. (1970a). The formation of aldehydes from amino acids by tea leaves extracts. Agric. Biol. Chem., 34, 227-233.

Saijo, R. and Takeo, T. (1970b). The production of phenylacetaldehyde from L-phenylalanine in tea fermentation. Agric. Biol. Chem., 34, 222-226.

Takeo, T. (1981). Production of linalool and geraniol by hydrolytic breakdown of bound forms in disrupted tea shoots. Phytochemistry, 20, 2145-2147.

Takeo, T. and Tsushida, T. (1980). Changes in lipoxygenase activity in relation to lipid degradation in plucked tea shoots. Phytochemistry, 19, 2521-2522.

Werkhoven, J. (1974) "Tea Processing." FAO Agricultural Services Bulletin, no 26, FAO, Rome.

Wicremasinghe, R.L., Wick, E.L. and Yamanishi, T. (1973). Gas chromatographic mass spectrophotometric analysis of flavoury and non-flavoury Ceylon black tea aroma concentrates prepared by different methods. J. Chromatgr., 79, 756-780.

Yamanishi, T., Botheju, W.S. and De Silva, J.M. (1989). An index for assessing the quality of Uva seasonal black teas. Sri Lanka J. Tea Sci. 58, 40-49.

Yamanishi, T., Kobayashi, A., Nakamura, H., Uchida, A., Mori, S., Ohsawa, K. and Sasakura, S. (1968). Flavor of black tea. V. Comparison of various black teas. Agric. Biol. Chem., 32, 379-386. Yamanishi, T., Wicremasinghe, R.L. and Perera, K.P.C.W. (1978) Studies on the flavor and quality of tea. 3-Gas chromatographic analysis of aroma complex. Tea $Q ., 39,81-86$. 